\title{
Etudes de l'activité anthelminthique et de la toxicité du dilaurate d'étain dibutyle chez le poulet
}

\author{
por M. GRABER ef G. GRAS
}

Le téniasis aviaire étant une maladie fort répandue sur tout le territoire de la République du Tchad, il importait de mettre au point une méthode de lutte valable contre ce parasitisme. Dans ce but, la section d'helminthologie du laboratoire de Farcha à Fort-Lamy a établi un programme de recherches en collaboration avec plusieurs laboratoires spécialisés dans l'étude des anthelminthiques. C'est dans le cadre de ces recherches, que l'étude du pouvoir anthelminthique de corps tels que le phloroglucinate de Diéthylène-diamine (GUILHON et GRABER 1961), I'Hexachlorophène (GUILHON 1961) et le Dichlorophène (GUILHON et GRABER) a été entreprise.

Outre ces dérivés de la piperazine et ces biphénols, les composés de l'étain ont retenu plus particulièrement notre attention. L'étude des composés minéraux et organiques de ce métal est poursuivie depuis plus de 4 ans en collaboration avec le laboratoire de pharmacie chimique de la faculté de Pharmacie de Montpellier (Pr. P. CASTEL). Ces recherches ont abouti à la mise au point d'un composé minéral très intéressant l'arséniate d'étain $\left(^{*}\right)$; ce produit tout en étant dépourvu de toxicité pour l'homme et pour les animaux, présente le gros avantage d'être polyvalent, c'est-à-dire actif à la fois sur les principaux Cestodes et sur les Ascaridia des volailles (CASTEL, GRABER, GRAS et CHHAY - HANCHENG, 1960). Il est également très actif sur l'ensemble des Cestodes intestinaux du mouton (CASTEL, GRABER, GRAS ef CHHAY-HANCHENG, 1960); son coefficient chimio-thérapique chez cet animal, le classe comme un des meilleurs anthelminthiques actuellement connus.

(*) Spécialisé sous le nom d'«ETANARSAN », laboratoires LATHEVET, 20, rue des Fossés Saint-Jacques, Paris XIe.

Rev. Elev. Med. vet. Pays trop. 1962, 15, no 4.

Reçu pour publication : Décembre 1962.
Les composés organiques de l'étain n'ont pas non plus été négligés. Ils sont connus depuis fort longtemps, mais, ils n'avaient reçu, en élevage aviaire, que peu d'applications pratiques avant 1941, époque où CUTHRIE et ses collaborateurs déterminèrent le pouvoir anthelminthique de certains d'entre eux : L'étain tétraéthyle, l'étain tétraphényle, l'étain tétraisobutyle' et le chlorure d'étain triphényle ; ces composés étaient soit trop toxiques, soit inactifs.

En 1951 et 1956, KERR et WALDE dans un très gros travail essayèrent plus de 150 composés organiques de l'étain sur des poulets expérimentalement infestés par Ascaridia galli et Raillietina cesticillus. Les composés essayés par ces auteurs étaient de quatre types principaux :

$$
\begin{array}{ll}
R_{4} \operatorname{Sn} & R_{2} \operatorname{Sn} X_{2} \\
R_{3} \operatorname{Sn} X & R \text { Sn } X_{3}
\end{array}
$$

$R$ représente un radical organique aryl ou alkyl ou aralkyl directement lié à l'atome d'étain par une liaison de covalence étain-carbone, $X$ est un anion simple ou complexe.

Ce sont les composés $R_{2} S_{n} X_{2}$ qui se sont montrés les plus efficaces, à condition: que $R$ et $X$ soient de structure relativement simple. Les composés $R_{3} S n X$ sont actifs mais trop toxiques. Quant aux dérivés $R S_{n} X_{3}$ et $R_{4} S n$, ils ne le sont que très peu ou pas du tout.

Dès 1952, KERR avait fait connaître un composé de type $R_{2} S n X_{2}$, le dilaurate d'étain dibutyle et, depuis cette époque, un grand nombre de publications font état de résultats favorables obtenus avec cet anthelminthique (BLOUNT 1955 ; EDGAR 1956 ; ABDOU 1956 ; WHITTEN 1956 ; EDGAR et TERR 1957 ; KERR ef WALDE 1956 ; TURK 1958 ; ENIGK et DUWEL 1959).

Cinq composés organiques de l'étain ont fait i l'objet de recherches, tant à la faculté de Phar- 
macie de Montpellier qu'au laboratoire de Farcha. Ce sont :

Le dilaurate d'étain dibutyle ;

le dichlorure d'étain di-n-octyle,

l'oxyde d'étain diphényle ;

le dichlorure d'étain diphényle ;

le maléate d'étain dibutyle.

Nous rapportons dans cette première note les résultats obtenus avec le dilaurate d'étain dibutyle.

\section{I, - DILAURATE D'ÉTAIN DIBUTYLE}

Caractéristiques ef propriétés physico-chimiques

La formule dé ce composé est la suivante:

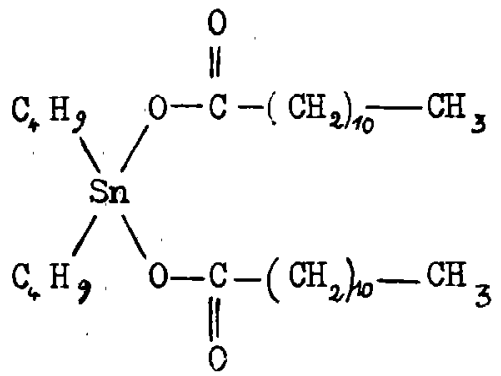

Le poids moléculaire est de 631,0 ; il titre 18,80 p. 100 en étain.

Le dilaurate d'étain dibutyle que pour simplifier nous nommerons DED, se présente sous forme d'une huile blanche ou légèrement jaune suivant le degré de pureté du produit. II possède une odeur aromatique caractéristique. En dessous de $15^{\circ} \mathrm{C}$, il se prend en masse. Les produits techniques sont en général préparés non à partir d'acide laurique pur mais de mélange d'acides gras provenant de l'huile de coco, ceci afin de donner au produit final un point de fusion inférieur à la température ordinaire, ce qui le rend plus facile à manipuler. La purification d'un tel produit ne se fait pas par distillation, mais par cristallisation partielle suivie de l'enlèvement de la partie liquide par préssurage.

Le produit que nous avons utilisé $\left(^{*}\right)$ est un produit préparé à partir de matières premières pures. Le dosage de l'étain effectué par la méthode de KOCHESHKOV a donné une teneur en étain de 18,72 p. 100 pour une teneur théorique de 18,80 p. 100 .

(*) Le produit a été préparé à l'Institut de chimie organique T. N. O. à Utrech, par le Docteur VAN DER KERK ef le Docteur LUIJTEN à qui nous adressons nos vifs remerciements. te DED est soluble à froid dans l'éther de pétrole, le benzène, l'acétone, le tétrachlorure de carbone et l'éther; il est insoluble dans l'eau et l'alcool méthylique.

Le DED est utilisé sur une grande échelle (SMITH 1952) comme stabilisant du chlorure de polyvinyle. La production mondiale pour cet usage est de plusieurs centaines de tonnes par an.

\section{II. - MATÉRIEL ET MÉTHODE}

\section{Matériel}

Les poulets ayant servi à l'expérimentation étaient originaires de la région ouest du Tchad (Fort-Lamy, Massakory et lac). Sur un nombre total de 136 animaux, 103 d'entre eux, soit 75,8 p. 100, hébergeaient des Nématodes et des Cestodes appartenant aux espèces suivantes:

$$
\begin{aligned}
\text { Cestodes : Choanotaenia infundibulum }: 5 \\
\\
\text { Raillietina tetragona : } 58 \\
\text { Raillietina echinobothrida : } 14 \\
\text { Raillietina cesticillus : } 14: \\
\text { Cotugnia digonopora : } 3 \\
\text { Hymenolepis carioca : } 4
\end{aligned}
$$

Nématodes': Strongyloïdes Sp: : 3

Ascaridia styphlocerca : 4

Subulura brumpti' : 47

Acuaria spiralis : 5

Dans 26 p. 100 des cas, ces dix espèces se trouvaient être associées chez le même animal en proportion variable :

Io Association à deux éléments : 22, soit $81 \mathrm{p}$. 100.

Raillietinatetragona + Raillietina echinobothrida:1

Raillietina tetragona + Raillietina cesticillus : 2

Raillietina fetragona + Subulura brumpti : 12

Raillietina tetragona + Acuaria spiralis : 1

Raillietina tetragona + Ascaridia styphlocerca : 1

Raillietina echinobothrida + Subulura brumpti : 1

Raillietina cesticillus + Subulura brumpti : 2

Strongyloides Sp. + Subulura brumpti : 1

Subulura brumpti + Acuaria spiralis : 1 100.

20 Association à trois éléments : 4, soit $15 \mathrm{p}$.

Raillietina tetragona + Raillietina echinobothrida +

Subulura brumpti : 2 ;

Raillietina fétragona + Choonotaenia infundibulum + Acuaria spiralis : 2. 


\begin{tabular}{|c|c|c|c|c|c|c|}
\hline \multicolumn{7}{|c|}{ TABLEAU NO I.-BUTYNORATE.-PARASITES AUUTTS.-DOSE JWIQUE.-DIETE DE 20 HEURES } \\
\hline $\begin{array}{r}\text { Doses } \\
(\mathrm{mg} / \mathrm{kg})\end{array}$ & $\begin{array}{l}\text { Nombre } d q \\
\text { animaux }\end{array}$ & $\begin{array}{l}\text { Poids des poulets } \\
\text { (en } \mathrm{g} \text { ) }\end{array}$ & Parasites on cause & $\begin{array}{l}\text { Nombre d'ani- } \\
\text { maux déparasités }\end{array}$ & efficacit & scolex \\
\hline 50 & $\begin{array}{l}1 \\
3\end{array}$ & $\begin{array}{l}1.000 \\
980 ; 1.000 ; 940\end{array}$ & $\begin{array}{l}\text { Raillietina tetragona } \\
\text { Subulura brunpti }\end{array}$ & $1 \underset{0}{\operatorname{sur}} 1$ & $\begin{array}{c}\text { Totale } \\
0\end{array}$ & 0 \\
\hline 80 & $\begin{array}{l}2 \\
1 \\
1 \\
2 \\
\end{array}$ & $\begin{array}{l}957 ; 885 \\
705 \\
1.342 \\
1.342 ; 607 \\
\end{array}$ & $\begin{array}{l}\text { Raillietina tetragona } \\
\text { Cotugnia digonopora } \\
\text { Hymenolepis carioca } \\
\text { Subulura brupti } \\
\end{array}$ & $\begin{array}{c}2 \text { sur } 2 \\
0 \\
0 \\
0\end{array}$ & $\begin{array}{l}\text { Totale } \\
\text { Nulle } \\
\text { Sulle } \\
\text { Nulle }\end{array}$ & $\begin{array}{c}0 \\
+++ \\
+++\end{array}$ \\
\hline 100 & $\begin{array}{r}10 \\
3 \\
1 \\
1 \\
1 \\
14\end{array}$ & $\begin{array}{l}680 ; 740 ; 540 ; 600 ; 600 \\
900 ; 700 ; 680 ; 580 ; 580 \\
677 ; 900 ; 560 \\
1.220 \\
900 \\
580 \\
677 ; 642 ; 740 ; 600 ; 900 \\
900 ; 700 ; 660 ; 640 ; 600 \\
540 ; 560 ; 560 ; 580\end{array}$ & $\begin{array}{l}\text { Raillietina tetragona } \\
\text { Raillietina echinobothrida } \\
\text { Raillietina cesticillus } \\
\text { Cotugnia digonopora } \\
\text { Ascaridia styphlocerca } \\
\text { Subulura brumpti }\end{array}$ & $\begin{array}{l}8 \text { sur } 10 \\
3 \text { sur } 3 \\
1 \text { sur } 1 \\
1 \text { sur } 1 \\
0 \\
0\end{array}$ & $\begin{array}{l}81.8 \% \\
\text { Totale } \\
\text { Totale } \\
\text { Totale } \\
\text { Nulle } \\
\text { Phulle }\end{array}$ & $\begin{array}{l}+ \\
0 \\
0 \\
0\end{array}$ \\
\hline 150 & $\begin{array}{l}1 \\
2 \\
1 \\
4 \\
1 \\
1 \\
6 \\
6 \\
3\end{array}$ & $\begin{array}{l}880 \\
880 ; 800 \\
980 \\
980 ; 800 ; 880 ; 900 \\
800 \\
880 \\
1.200 ; 900 ; 760 ; 800 \\
820 ; 860 \\
640 ; 1.200 ; 860\end{array}$ & $\begin{array}{l}\text { Raillietina tetragona } \\
\text { Raillietina echinobothrida } \\
\text { Raillietina cesticillus } \\
\text { Subulura brumpti } \\
\text { Acuaria spiralis } \\
\text { Ascaridia styphlocerca } \\
\text { Raillietina tetragona } \\
\text { Raillietina cesticillus }\end{array}$ & $\begin{array}{l}1 \text { sur } 1 \\
2 \operatorname{sur} 2 \\
1 \text { sur } 1 \\
0 \\
0 \\
0 \\
6 \operatorname{sur} 6 \\
3 \operatorname{sur} 3 \\
\end{array}$ & $\begin{array}{l}\text { Totale } \\
\text { Totale } \\
\text { Totale } \\
\text { Nulle } \\
\text { Nulle } \\
\text { Nulle } \\
\text { Totale } \\
\text { Totale } \\
\end{array}$ & $\begin{array}{l}0 \\
0\end{array}$ \\
\hline 200 & $\begin{array}{l}6 \\
4 \\
1 \\
i \\
7\end{array}$ & $\begin{array}{l}500 ; 500 ; 520 ; 629 ; 642 \\
457 \\
564 ; 660 ; 490 ; 560 \\
659 \\
500 \\
520 ; 642 ; 457 ; 490 ; 560 \\
560 ; 817 \\
\end{array}$ & $\begin{array}{l}\text { Raillietina tetragona } \\
\text { Raillietina echinobothrida } \\
\text { Raillietina cesticillus } \\
\text { Cotugnia digonopora } \\
\text { Subulura brumpti }\end{array}$ & $\begin{array}{l}6 \text { sur } 6 \\
4 \text { sur } 4 \\
1 \text { sur } 1 \\
1 \underset{0}{\operatorname{sur}} 1\end{array}$ & $\begin{array}{l}\text { Totale } \\
\text { Totale } \\
\text { Totale } \\
\text { Totale } \\
\text { Nulle }\end{array}$ & $\begin{array}{l}0 \\
0 \\
0 \\
0\end{array}$ \\
\hline 250 & $\begin{array}{l}2 \\
2 \\
2\end{array}$ & $\begin{array}{l}760 ; 760 \\
755 ; 440 \\
755 ; 440\end{array}$ & $\begin{array}{l}\text { Raillietina tetragona } \\
\text { Stro-gloides Sp. } \\
\text { Subulura brumpti }\end{array}$ & $\begin{array}{c}2 \operatorname{sur} \\
0 \\
0\end{array}$ & $\begin{array}{l}\text { Totale } \\
\text { Nulle } \\
\text { Inulle }\end{array}$ & \\
\hline 300 & $\begin{array}{l}1 \\
1\end{array}$ & $\begin{array}{l}1.120 \\
690\end{array}$ & $\begin{array}{l}\text { Raillietina tetragona } \\
\text { Subulura brunpti }\end{array}$ & $1 \operatorname{sur}_{0} 1$ & $\begin{array}{l}\text { Totale } \\
\text { Thulle }\end{array}$ & $0^{\prime}$ \\
\hline 400 & $\begin{array}{l}1 \\
1 \\
\end{array}$ & $\begin{array}{l}700 \\
700 \\
\end{array}$ & $\begin{array}{l}\text { Raillietina tetragona } \\
\text { Subulura brumpti }\end{array}$ & $\begin{array}{c}1 \operatorname{sur}_{1}^{1} \\
0\end{array}$ & $\begin{array}{l}\text { Totale } \\
\text { Shulle. }\end{array}$ & 0 \\
\hline 500 & $\begin{array}{l}5 \\
1 \\
2 \\
3\end{array}$ & $\begin{array}{l}710 ; 774 ; 610 ; 620 \\
660 \\
600 \\
700 ; 660 \\
600 ; 660 ; 640\end{array}$ & $\begin{array}{l}\text { Raillietina tetragona } \\
\text { Raillietina echinobothrida } \\
\text { Raillietina cesticillus } \\
\text { Subulura brumpti. }\end{array}$ & $\begin{array}{c}5 \text { sur } 5 \\
1 \text { sur } 1 \\
2 \text { sur } 2 \\
0\end{array}$ & $\begin{array}{l}\text { Totale } \\
\text { Totale } \\
\text { Totale } \\
\text { Nulle }\end{array}$ & $\begin{array}{l}0 \\
0 \\
0\end{array}$ \\
\hline 800 & $\begin{array}{l}2 \\
1\end{array}$ & $\begin{array}{l}600: 620 \\
600\end{array}$ & $\begin{array}{l}\text { Raillietina tetragona } \\
\text { Raillietina echinobothrida }\end{array}$ & 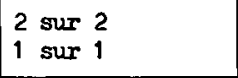 & $\begin{array}{l}\text { Totale } \\
\text { Totale }\end{array}$ & $\begin{array}{l}0 \\
0\end{array}$ \\
\hline 1000 & $\begin{array}{l}3 \\
1\end{array}$ & $\begin{array}{l}580 ; 500 ; 520 \\
590\end{array}$ & $\begin{array}{l}\text { Raillietina tetragona } \\
\text { Raillietina cesticillus }\end{array}$ & $\begin{array}{l}3 \text { sur } 3 \\
1 \text { sur } 1\end{array}$ & $\begin{array}{l}\text { Totale } \\
\text { Totale }\end{array}$ & $\begin{array}{l}0 \\
0\end{array}$ \\
\hline 1200 & 2 & $580 ; 580$ & Raillietina tetragona & 2 sur 2 & Totale & 0 \\
\hline 1500 & $\begin{array}{l}3 \\
1 \\
1 \\
2\end{array}$ & $\begin{array}{l}540 ; 500 ; 540 \\
520 \\
560 \\
500 ; 540\end{array}$ & $\begin{array}{l}\text { Raillietina tetragona } \\
\text { Ascaridia styphlocerca } \\
\text { Acuaris spiralis } \\
\text { Subulura brumpti }\end{array}$ & $\begin{array}{c}3 \text { sur } 3 \\
0 \\
0 \\
0\end{array}$ & $\begin{array}{l}\text { Totale } \\
\text { Nulle } \\
\text { Nulle } \\
\text { Nulle }\end{array}$ & 0 \\
\hline
\end{tabular}


$3^{\circ}$ Associatian à quatre éléments : 1 , soił 4 p. 100. $R$. tétragona $+R$. echinobothrida + Ascaridia styphlocerca + Subulura brumpti : 1 .

Ce lot de poulet - moins intéressant que les précédents, parce que beaucoup moins parasitéa néanmoins permis de se faire une idée de la polyvalence éventuelle du DED.

\section{Epoque.}

Les essais ont eu lieu en quatre temps : juinjuillet 1960 ; novembre-décembre 1960 ; févrieravril-mars 1961 et septembre-octobre 1961. De cette façon, il a été possible d'apprécier l'action de l'antheiminthique en période favorable (fin de l'été, automne, hiver) où les animaux trouvent à se nourrir abondamment, et en période défavorable (fin de l'hiver-printemps et début de l'été).

\section{Technique.}

C'cst unc technique de portéc génćrale absolument comparable d̀ celle qui a été employée précédemment lors des essais avec l'arséniate d'étain (CASTEL, GRABER, GRAS et CHHAY-HANCHENG, 1960). Nous la rappellerons donc brièvement : dans un premier temps, chaque animal a été mis au repos durant 36 heures environ, de manière à libérer tous les parasites susceptibles de s'éliminer naturellement sans aucune intervention.

Les oiseaux ont été placés dans des cages grilla gées, sur des supports de bois, à $25 \mathrm{~cm}$ du sol et les excréments recueillis sur des plateaux disposés au-dessous, de façon à empêcher l'absorption par des poulets coprophages des parasites expulsés au cours de l'expérience.

Les animaux ont éfé soumis à une diète complète de 20 heures avant et une heure après l'administration de l'anthelminthique.

Les doses uniques ont été d'abord calculées en fonction du poids de chaque volatile et introduites dans des capsules de gélatine de type auréomycine. Par la suite, il n'a plus été tenu compte du poids et une dose unique de $125 \mathrm{mg}$ a été donnée uniformément à chaque animal.

Après traitement, les excréments ont été ramassés, broyés dans de l'eau et minutieusement examinés, dans le but de prélever les parasites évacués.

Au bout d'un temps variable - 5 à 10 jours - les animaux ont été sacrifiés ef l'intestin visité complètement. Les premières portions ont été grattées sur une distance d'environ $25 \mathrm{~cm}$ et il a été procédé à 2 ou 3 examens du produit de raclage entre lame et lamelle. Cette technique est absolument indispensable pour retrouver les formes jeunes immatures, les scolex de Choanotaenia infundibulum et de Raillietina qui demeurent « in situ », bien que leurs chaînes aient cédé à l'action du ténifuge et Hyménolepis carioca qui est toujours profondément englobé dans le mucus de l'intestin.

Les Cestodes récoltés dans les excréments après traitement et ceux découverts après autopsie ont été pesés séparément. La' comparaison entre ce qui est chassé et ce qui reste, apporte la preuve de l'efficacité du produit, comple tenu des résultats fournis par le grattage des muqueuses.

\section{III. - RESULTATS}

\section{Action sur les Cestodes}

a) Formes adultes.

Les résultats sont rapportés dans les tableaux I, II, III.

b) Formes immatures.

Les résultats sont rapportés dans le tableau IV.

Dans les conditions du Tchad, le DED, à la dose de $125 \mathrm{mg} / \mathrm{kg}$ provoque l'élimination totale des formes adultes de Raillietina tetragona, Raillietina echinobothrida, Raillietina cesticillus et Cotugnia digonopora. Les doses supérieures ne font pas preuve d'une meilleure activité. Quant aux doses inférieures à $125 \mathrm{mg} / \mathrm{kg}$, elles sont irrégulières.

A $100 \mathrm{mg} / \mathrm{kg}$, l'expulsion, de Raillietina cesticillus, Raillietina echinobothrida ef Cotugnia digonopora semble complète ; par contre, le pourcentage de réduction n'atteint que 81 p. 100 sur Raillietina tetragona.

A $80 \mathrm{mg} / \mathrm{kg}$. l'action est nulle sur Hymenolepis carioca et sur Cotugnia digonopora, excellente sur Raillietina tetragona.

Les formes immatures sont absolument toutes détruites au delà de $200 \mathrm{mg} / \mathrm{kg}$. Au-dessous, un certain nombre de formes immatures de Raillietina tetragona, Raillietina echinobothrida et Raillietina cesticillus demeurent présentes dans les proportions suivantes :

2 animaux sur 6 à $80 \mathrm{mg} / \mathrm{kg}$

4 animaux sur 30 à $100 \mathrm{mg} / \mathrm{kg}$

1 animal sur 16 à $200 \mathrm{mg} / \mathrm{kg}$.

Plus les doses augmentent, plus le nombre de formes immatures diminue. Vers $100 \mathrm{mg} / \mathrm{kg}$, un 
animal sur 10 environ est encore porteur de Cestodes après administration de DED. Ce point méritait d'être souligné : un bon ténifuge doit permettre en effet l'évacuation totale à la fois des formes adultes et des formes immatures et le DED
1 - les formes adultes de Raillietina tetragona, de Raillietina echinobothrida, de Cotugnia digonopora et sans doute d'Hymenolepis carioca sont à peu près toutes détruites à la dose unique de 100 $\mathrm{mg} / \mathrm{kg}$.

\begin{tabular}{|c|c|c|c|c|c|}
\hline \multirow[b]{2}{*}{$\begin{array}{l}\text { Nowbre d' } \\
\text { animaux }\end{array}$} & BUTYNORATE.-PARASITES & $\begin{array}{c}\text { TABLEAU } \\
\text { ASITES ADULTES,-DOS } \\
\text { DIETE DE } 20\end{array}$ & \multicolumn{3}{|c|}{$\begin{array}{l}\text { ADULTES, -DOSE UNIQUE DE } 125 \text { MG PAR TERE } \\
\text { DIETE DE } 20 \text { HEURES }\end{array}$} \\
\hline & $\begin{array}{c}\text { Poids des } \\
\text { poulets(en E) }\end{array}$ & Parasites en cause & $\begin{array}{c}\text { Nombre d'ani- } \\
\text { nawx déparasités }\end{array}$ & Efficacité & $\begin{array}{l}\text { Soo- } \\
\text { lex }\end{array}$ \\
\hline 3 & $660 ; 700 ; 700$ & $\begin{array}{l}\text { Raillietina te- } \\
\text { tragone }\end{array}$ & $3 \operatorname{sur} 3$ & Totale & 0 \\
\hline 2 & $740 ; 760$ & $\begin{array}{l}\text { Choanotaenia } \\
\text { infundibulum }\end{array}$ & 2 sur 2 & Totale & 0 \\
\hline 1 & 980 & $\begin{array}{l}\text { Raillietina } \\
\text { echinobottrida }\end{array}$ & 1 sur 1 & Totale & 0 \\
\hline 1 & 600 & $\begin{array}{l}\text { Subulura } \\
\text { brumpti }\end{array}$ & 0 & Nulie & 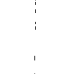 \\
\hline
\end{tabular}

ne paraît pas répondre dans fous les cas à cette double vocation. L'ensemble des résultats recueillis au Tchad figure au tableau no $\mathrm{V}$.

Le DED a été expérimenté dans de nombreux pays sur à peu près tous les Cestodes aviaires les plus communs. Le tableau no $\mathrm{V} /$ résume les principaux travaux effectués depuis 10 ans.

De toutes ces expérimentations, il est possible de tirer les conclusions suivantes:

\begin{tabular}{|c|c|c|}
\hline \multicolumn{3}{|c|}{$\begin{array}{l}\text { TABLEAD III } \\
\text { TEMOINS }\end{array}$} \\
\hline Juin-Juillet 1960 & $\begin{array}{c}\text { Fóvrier_Harg-Avril } \\
1961\end{array}$ & Septembre 1961 \\
\hline 6 anjingux & 5 antangx & 5 aningux \\
\hline $\begin{array}{l}\text { Raillietina } \\
\text { tetragona : } 1 \mathrm{~g} .\end{array}$ & R. tetragona $: 1,6 \mathrm{~g}$. & $\begin{array}{l}\text { Choen. Infundi- } \\
\text { bulum : } 0,1 \mathrm{~g} \text {. }\end{array}$ \\
\hline $\begin{array}{l}\text { R. echinobo- } \\
\text { thrida: } 1,5 \mathrm{gs} .\end{array}$ & $\begin{array}{l}\text { Hypenolepia } \\
\text { carioca : 0,2 } 8 \text {. }\end{array}$ & $\begin{array}{l}\text { R. tetra- } \\
\text { gana : } 0,9 \mathrm{~g} \text {. }\end{array}$ \\
\hline $\begin{array}{l}\text { R. cesticilllus : } \\
0,6 \mathrm{~g} \text {. }\end{array}$ & $\begin{array}{l}\text { R. costicillus : } \\
3 \mathrm{~g} .\end{array}$ & \\
\hline $\begin{array}{l}\text { Acuaria spiralis : } \\
\text { to g. } \\
\text { Subulura brumpti : } \\
15 \mathrm{~g} \text {. }\end{array}$ & $\begin{array}{l}\text { Subulure } \\
\text { brumpti : } 13 \mathrm{~g} .\end{array}$ & $\begin{array}{l}\text { Subulura } \\
\text { brumpti : } 21 \mathrm{E} .\end{array}$ \\
\hline \multicolumn{3}{|c|}{$\begin{array}{l}\text { Ghoan. = Choanotaenia } \\
R_{1}=\text { Railltetina }\end{array}$} \\
\hline
\end{tabular}

$2^{\circ}$ Pour Choanotoenia infundibulum et Raillietina cesticillus, $125 \mathrm{mg} / \mathrm{kg}$ constifue une dose limite inférieure au-dessous de laquelle les résultats risquent d'être très irréguliers.

3o Sur Davainea proglottina et. Amoebotaenia sphenoïdes, les avis divergent : pour ENIGK et DUWEL (1959), les doses inférieures à $188 \mathrm{mg} /$ $\mathrm{kg}$ sont suffisantes, tandis qu'EDGAR (1956) se montre très satisfait de la dose de $125 \mathrm{mg} / \mathrm{kg}$. Les deux parasites n'existant pas au 'Tchad, aucun essci n'a donc pu être tenté sur ces deux Cestodes.

En Afrique centrale, avec des poulets dont le poids variait de 480 à $1.000 \mathrm{~g}$, la dose de $125 \mathrm{mg}$ par tête de DED s'est révélée suffisamment intéressante pour être préconisée : l'essai réalisé portait sur un lot de 2 animaux dont le poids moyen était de $690 \mathrm{~g}$. ce qui représente $180 \mathrm{mg}$ de DED par $\mathrm{kg}$ de poids vif. II est possible de détruire ainsi toutes les formes adultes ef la plupart des formes immatures des, six Cestodes aviaires les plus fréquents au Tchad.

\section{Action sur les Nématodes}

Elle est absolument nulle dans tous les cas, que ce soit sur Ascaridia styphloccrca de l'intestin, Subulura brumpti des caecums, Strangyloides sp. de I'intestin ou Acuaria spiralis du ventricule succenturié. 
La monovalence du DED enlève à cet anthelminthique une grande partie de sa valeur puisque I'on connaît actuellement - et c'est le cas pour l'arséniate d'étain - des ténifuges aviaires polyvalents, c'est-à-dire actifs à la fois sur la plupart des Cestodes adultes ou immatures et sur les Ascaridia du poulet. n'est pas connu, toutefois, on peut penser que ce type de composé agit en bloquant les anti-diastases qui permettent aux vers de se maintenir dans le tube digestif sans être digérés. Une fois ce système bloqué, le parasite est soumis à l'action dissolvante des sucs intestinaux et notamment de la bile; le ver est alors digéré plus ou moins

\begin{tabular}{|c|c|c|c|c|}
\hline \multicolumn{4}{|c|}{$\begin{array}{c}\text { TABLAAUU IV } \\
\text { DED.- Parasites immatures.- Diète de } 20 \text { heures }\end{array}$} & \multirow[b]{2}{*}{$\begin{array}{c}\text { Témoing }+ \\
\text { (formes immatures) }\end{array}$} \\
\hline $\begin{array}{c}\text { Doses en } \\
(\mathrm{mg} / \mathrm{kg})\end{array}$ & $\begin{array}{l}\text { Nombre de } \\
\text { poulets } \\
\text { traités }\end{array}$ & $\begin{array}{c}\text { Nombre de } \\
\text { poulets } \\
\text { encore } \\
\text { parasités }\end{array}$ & Parasites en cause + & \\
\hline 80 & $\begin{array}{l}6 \\
6\end{array}$ & $\begin{array}{l}1 \\
1\end{array}$ & $\begin{array}{l}\text { Raillietina tetragona : } 1 \\
\text { R. echinobothrida : } 1\end{array}$ & $\begin{array}{l}\quad 10 \text { animaux } \\
\text { Choan. Infund: : } 3 \\
\text { R. tet. : } 5 \\
\text { R. cest. : } 8 \text { : } \\
\text { R. echinob. : } 2\end{array}$ \\
\hline \multirow[t]{2}{*}{100} & 30 & 2 & $\begin{array}{l}\text { Raillietina tetragona : } 2 \\
\text { Raillietina cesticillus: } 1\end{array}$ & idem \\
\hline & 16 & 1 & Raillietina tetragona : 1 & $\begin{array}{l}\quad 6 \text { animaux } \\
\text { Choan. Infund. : } 2 \\
\text { R. tet : } 5 \text {, } \\
\text { R. cest : } 7 \\
\text { Hym. car. }\end{array}$ \\
\hline & $\begin{array}{l}\text { Choan. }+ \\
\mathrm{R} \text {, echin } \\
\text { Hym. car } \\
\mathrm{R} \text {. tet. } \\
\mathrm{R} \text {. cest. }\end{array}$ & $\begin{array}{l}=\text { Nombre } \\
\text { nfund }=\text { Ch } \\
10 b=\text { Raill } \\
=\text { = Hymeno } \\
=\text { Railliet } \\
=\text { Raillie }\end{array}$ & $\begin{array}{l}\text { de parasites (moyenne) } \\
\text { loariotaenia infundibulum } \\
\text { letina echinobothrida. } \\
\text { lepis carioce } \\
\text { ina tetragona } \\
\text { tina cesticillus }\end{array}$ & : \\
\hline
\end{tabular}

\section{IV. - MODE D'ACTION}

Le DED agit rapidement sur les parasites qui sont éliminés en une seule fois, généralement 24 h. après l'administration du produit. II est rare que ce délai soit dépassé.

Les Cestodes sont expulsés sous forme de petits fragments déjà en partie digérés. Le mécanismc intime par lequel le DED agit sur les Cestodes rapidement ; les proglottis șitués dans la dernière partie du tube digestif moins sensibles par leur position sont à peu près seuls à être rejetés dans le milieu extérieur. Ces fragments dont une partie de la cuticule est dissoute sont alors facilement reconnaissables après éclaircissement ou coloration.

Le DED agit done plus comme un ténicide que comme un ténifuge. 


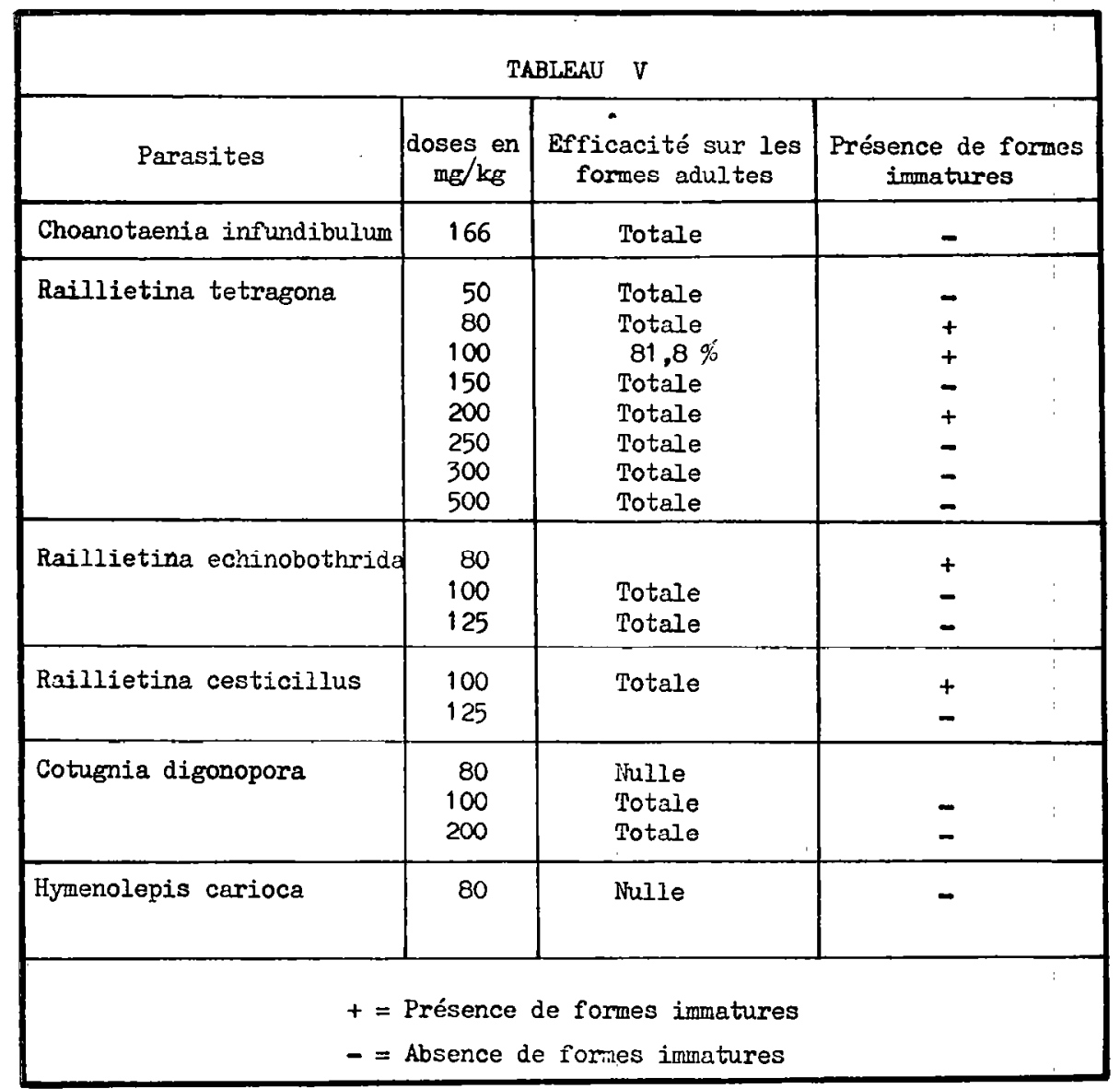

\section{V. - CONSÉQUENCES DU TRAITEMENT}

\section{Sur l'animal.}

Elles sont faibles à la dose de $125 \mathrm{mg}$ par tête. II n'y a que peu de changements dans l'attitude et le comportement extérieur des volailles.

Les modifications éventuelles des éléments du sang à la suite du traitement au DED ont été suivies pendant plusieurs jours sur divers lots de poulets soumis à différentes doses : Les résultats sont rapportés dans le tableau VII.

Après traitement au DED, on observe, sur des poulets normalement entretenus, une augmentation du nombre des hématies et, dans certains cas, des lymphocytes et des polynucléaires neutrophiles. Quant aux leucocytes, ils ne subissent pratiquement pas de variations, quelle que soit la dose. Le taux d'éosinophiles baisse en général, ce qui est normal puisque les poulets ont été débarrassés des Cestodes qui sont en partie responsables de l'accroissement notable de nombre je ces polynucléaires.

\section{Augmentation de poids.}

Elle a été signalée par KERR (1952) : elle serait plus importante à la cinquième semaine faisant suite au traitement qu'à la neuvième.

Le neuvième jour après l'administration de l'anthelminthique, les résultats enregistrés à Fort-Lamy sont très variables;

De 8 à 11 p. 100 d'augmentation de poids sur deux lots de 3 et 4 poulets (100 et $200 \mathrm{mg} / \mathrm{kg}$ ).

Une perte de poids de 5 p. 100 sur les 11 animaux ayant reçu $125 \mathrm{mg}$ par tête.

30 Répercussion sur la ponte.

Aucun essai n'a été effectué sur place. D'après KERR (1952), à la dose de $177 \mathrm{mg} / \mathrm{kg}$, qui corres- 


\begin{tabular}{|c|c|c|c|c|}
\hline \multicolumn{5}{|c|}{ TABLEAIS VI } \\
\hline Auteur & Parasites & Pays & $\begin{array}{l}\text { Doses } \\
(\mathrm{mg} / \mathrm{kg})\end{array}$ & $\begin{array}{c}\text { Efficacité } \\
(p \cdot 100)\end{array}$ \\
\hline KERR 1952 & Raillietina cesticillus & $\begin{array}{c}\text { U.S.A. } \\
\text { " } \\
" \\
" \\
" \\
"\end{array}$ & $\begin{array}{rr}50 & (\mathrm{~A}) \\
75 & (\mathrm{~A}) \\
100 & (\mathrm{~A}) \\
150 & (\mathrm{~A}) \\
200 & (\mathrm{~A}) \\
300 & (\mathrm{~A})\end{array}$ & $\begin{array}{r}32 \\
86 \\
92 \\
100 \\
88\end{array}$ \\
\hline BLOUNT 1955 & Raillietina Sp. & Angleterre & 75 (A) & totale \\
\hline EDGAR 1956 & $\begin{array}{l}\text { Raillietina cesticillus } \\
\text { Choanotaenia infundi- } \\
\text { bulum } \\
\text {-idem- } \\
\text { Davainea proglottina } \\
\text { Hymenolepis carioca } \\
\text { RaiJlietina tetragona } \\
\text { Amoebotaenia sphenoides }\end{array}$ & $\begin{array}{c}\text { U.S.A. } \\
" 1 \\
" 1 \\
" \\
" \\
"\end{array}$ & $\begin{array}{r}75(\mathrm{~A}) \\
75-100(\mathrm{~A}) \\
100(\mathrm{~A}) \\
108-125(\mathrm{~A}) \\
75(\mathrm{~B}) \\
125(\mathrm{~A}) \\
125(\mathrm{~A})\end{array}$ & $\begin{array}{l}\text { totale } \\
66-100 \\
\text { totale } \\
85-100 \\
\text { totale } \\
\text { totale } \\
\text { totale }\end{array}$ \\
\hline ABDOU 1957 & Davainea proglottina & Angleterre & $0,5-1 c c^{*}$ & totale \\
\hline EDGER et IERh & $\begin{array}{l}\text { Raillietina cesticillus } \\
\text {-idem- } \\
\text { Choanotaenia infundi- } \\
\text { bulum } \\
\quad \text {-iden- }\end{array}$ & $\begin{array}{c}\text { U.S.A. } \\
n \\
n \\
n\end{array}$ & $\begin{array}{l}42(A) \\
75(A) \\
25(B) \\
50(A)\end{array}$ & $\begin{array}{r}94 \\
\text { totale } \\
88 \\
\text { totale }\end{array}$ \\
\hline $\begin{array}{l}\text { ENIGK et } \\
\text { DUWEL }\end{array}$ & $\begin{array}{l}\text { Raillietina cesticillus } \\
\text {-idem- } \\
\text { Raillietina cesticillus } \\
\text { Choanotaenia infundi- } \\
\text { bulum } \\
\text {-idem- } \\
\text { Hyuenolepis carioca } \\
\text {-idem- } \\
\text { Devainea proglottina } \\
\text {-idem- } \\
\text {-idem- } \\
\text {-idem- } \\
\text { Amoebotaenia sphenoides }\end{array}$ & $\begin{array}{c}\text { Allemagne } \\
\text { " } \\
\text { " } \\
\text { " } \\
\text { " } \\
\text { " } \\
\text { " } \\
\text { " } \\
\text { " }\end{array}$ & $\begin{array}{r}125(\mathrm{~A}) \\
188(\mathrm{~A}) \\
282(\mathrm{~A}) \\
188(\mathrm{~A}) \\
282(\mathrm{~A}) \\
188(\mathrm{~A}) \\
282(\mathrm{~A}) \\
94(\mathrm{~A}) \\
188(\mathrm{~A}) \\
282(\mathrm{~A}) \\
376(\mathrm{~A}) \\
125(\mathrm{~A})\end{array}$ & $\begin{array}{l}90 \\
\text { totale } \\
\text { totale } \\
\text { totale } \\
\text { totile } \\
\text { totale } \\
\text { totale } \\
\text { nulle } \\
\text { nulle } \\
\text { totale } \\
\text { totale } \\
\text { nulle }\end{array}$ \\
\hline $\begin{aligned} A= & \text { Dose unt } \\
B= & \text { Dose rép } \\
*= & \text { Préparat } \\
& \text { est inco } \\
* *= & \text { Wormal }\end{aligned}$ & $\begin{array}{l}\text { que } \\
\text { étée deux fois à } 24 \text { heures } \\
\text { ion où la quantité de DED } \\
\text { anue }\end{array}$ & $\begin{array}{l}\text { d'interval } \\
\text { mise en sus }\end{array}$ & le & \\
\hline
\end{tabular}


pond à celle recommandée au Tchad, il n'existe aucune modification sensible de la production journalière d'œufs.

Par contre, EDGAR (1956), toujours à la même dose, note une baisse de production qui redevient normale 8 à 10 jours après la fin du traitement.

Il en est de même des nombreuses préparations dont il sera question plus loin.

La dose de $75 \mathrm{mg} / \mathrm{kg}$ est sans effet marqué sur la ponte.

\section{VI. - MODE D'ADMINISTRATION}

Dans notre expérimentation nous avons administré le DED en capsules de gélatine rigoureusement dosées. Les auteurs américains (KERR 1952 ef EDGAR 1956) utilisent l'anthelminthique soit en capsules, soit mélangé à la nourriture dans des proportions variables : 0,02 p. 100 pendant 15 semaines; 0,01 p. 100 pendant 13 semaines ou $500 \mathrm{mg}$ par $\mathrm{kg}$ de nourriture pendant 96 heures.

D'autres préparations ont été conseillées dont

\begin{tabular}{|c|c|c|c|c|c|c|c|c|}
\hline & & & TABLEAII & VII & & & & \\
\hline $\begin{array}{r}\text { Dose } \\
\mathrm{mg} / \mathrm{kg}\end{array}$ & Date & Hématieo & $\begin{array}{l}\text { Leuco- } \\
\text { cytes }\end{array}$ & $\begin{array}{c}\text { Lympho- } \\
\text { cytes }\end{array}$ & $\begin{array}{l}\text { Mono- } \\
\text { cytes }\end{array}$ & $\begin{array}{l}\text { Neutro- } \\
\text { philes }\end{array}$ & $\begin{array}{l}\text { Eosino- } \\
\text { philes }\end{array}$ & $\begin{array}{l}\text { Baso- } \\
\text { philes }\end{array}$ \\
\hline \multirow{3}{*}{150} & $\begin{array}{l}\text { Poulet } 1 \\
20-9-1961 \\
20-9-1961 \\
23-9-1961 \\
24-9-1961 \\
25-9-1961\end{array}$ & $\begin{array}{l}3.600 .000 \\
3.725 .000 \\
3.800 .000 \\
4.050 .000 \\
4.750 .000\end{array}$ & $\begin{array}{r}10.200 \\
9.800 \\
9.000 \\
10.000 \\
11.000\end{array}$ & $\begin{array}{l}72 \\
77 \\
53 \\
62 \\
65\end{array}$ & $\begin{array}{l}0 \\
0 \\
0 \\
1 \\
0\end{array}$ & $\begin{array}{l}17 \\
12 \\
36 \\
28 \\
24\end{array}$ & $\begin{array}{r}11 \\
11 \\
11 \\
9 \\
11\end{array}$ & $\begin{array}{l:}0 \\
0\end{array}$ \\
\hline & $\begin{array}{l}\text { Poulet } 2 \\
20-9-1961 \\
21-9-1961 \\
23-9-1961 \\
25-9-1961\end{array}$ & $\begin{array}{l}3.125 .000 \\
3.400 .000 \\
3.500 .000 \\
3.750 .000\end{array}$ & $\begin{array}{r}8.400 \\
8.800 \\
11.000 \\
10.000\end{array}$ & $\begin{array}{l}75 \\
77 \\
79 \\
77\end{array}$ & $\begin{array}{l}0 \\
0 \\
0 \\
0\end{array}$ & $\begin{array}{l}16 \\
13 \\
14 \\
17\end{array}$ & $\begin{array}{r}9 \\
10 \\
7 \\
6\end{array}$ & $\begin{array}{l:l} & \\
& 1 \\
0 & \\
0 & \vdots \\
0 & \\
0 & \end{array}$ \\
\hline & $\begin{array}{l}\text { Poulet } 3 \\
20-9-1961 \\
21-9-1961 \\
23-9-1961 \\
25-9-1961\end{array}$ & $\begin{array}{l}3.300 .000 \\
3.700 .000 \\
3.725 .000 \\
3.950 .000\end{array}$ & $\begin{array}{r}9.200 \\
9.000 \\
8.000 \\
11.000\end{array}$ & $\begin{array}{l}77 \\
76 \\
85 \\
80\end{array}$ & $\begin{array}{l}0 \\
0 \\
0 \\
0\end{array}$ & $\begin{array}{r}12 \\
10 \\
9 \\
14\end{array}$ & $\begin{array}{r}11 \\
14 \\
6 \\
6\end{array}$ & $\begin{array}{l}0 \\
0 \\
0 \\
0\end{array}$ \\
\hline 100 & $\begin{array}{r}\text { Poulet } 4 \\
7-9-1961 \\
8-9-1961 \\
9-9-1961 \\
12-9-1961\end{array}$ & $\begin{array}{l}3.700 .000 \\
4.500 .000 \\
4.225 .000 \\
4.150 .000\end{array}$ & $\begin{array}{l}8.400 \\
9.000 \\
9.000 \\
8.800\end{array}$ & $\begin{array}{l}52 \\
60 \\
57 \\
71\end{array}$ & $\begin{array}{l}0 \\
0 \\
1 \\
0\end{array}$ & $\begin{array}{l}22 \\
24 \\
23 \\
24\end{array}$ & $\begin{array}{r}26 \\
16 \\
19 \\
5\end{array}$ & $\begin{array}{l}0 \\
0 \\
0 \\
0\end{array}$ \\
\hline 400 & $\begin{array}{l}\text { Poulet } 5 \\
20-9-1961 \\
21-9-1961 \\
23-9-1961 \\
25-9-1961\end{array}$ & $\begin{array}{l}4.650 .000 \\
4.725 .000 \\
4.875 .000 \\
4.500 .000\end{array}$ & $\begin{array}{l}10.200 \\
11.600 \\
11.500 \\
11.000\end{array}$ & $\begin{array}{l}72 \\
80 \\
73 \\
70\end{array}$ & $\begin{array}{l}0 \\
1 \\
0 \\
1\end{array}$ & $\begin{array}{r}15 \\
6 \\
6 \\
14\end{array}$ & $\begin{array}{l}13 \\
13 \\
21 \\
15\end{array}$ & $\begin{array}{l:}0 \\
0\end{array}$ \\
\hline 800 & $\begin{array}{l}\text { Poulet } 6 \\
20-9-1961 \\
23-9-1961 \\
29-9-1961\end{array}$ & $\begin{array}{l}3.100 .000 \\
3.100 .000 \\
3.900 .000\end{array}$ & $\begin{array}{l}12.000 \\
10.500 \\
12.000\end{array}$ & $\begin{array}{l}69 \\
77 \\
75\end{array}$ & $\begin{array}{l}0 \\
1 \\
2\end{array}$ & $\begin{array}{r}13 \\
7 \\
12\end{array}$ & $\begin{array}{l}18 \\
15 \\
11\end{array}$ & 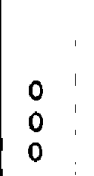 \\
\hline
\end{tabular}


le «Wormal » (*) qui renferme $125 \mathrm{mg}$ de DED, $22 \mathrm{mg}$ de nicotine et $500 \mathrm{mg}$ de phenothiazine. L'«Omnivermyl» (**) paraît être une préparation voisine. II est bon de signaler que ENIGK et DUWEL ont constaté que le «Wormal »'a qu'une action limitée sur Davaiena proglottina ef sur Amoebataenia, sphenoides. Dans tous les cas, quelle que soit la forme sous laquelle l'anthelminthique est distribué il vaut mieux mettre les animaux à la diète une vingtaine d'heures avant et 3 heures après le traitement.

\section{VII. - TOXICITÉ}

L'étude de la toxicité du DED a été envisagée sous deux aspects. Tout d'abord, nous avons étudié la toxicité du DED pour le poulet. Le médicament devant être administré en une seule dose ef par voie orale, seule la toxicité aiguë a été déterminée par cette voie d'administration.

Nous avons envisagé ensuite les possibilités de présence du DED, dans les parties des volailles destinées à la consommation. Ce deuxième aspect de la toxicité du DED, qui n'est qu'un cas pariiculier de la contamination des matières alimentaires est très important puisqu'il est directement en relation avec la santé du consommateur.

Io Toxicité du dilaurate d'élain dibutyle pour le poulet.

La toxicité du DED a été étudiée dès 1952 par KERR. Cet auteur remarque que, chez le poulet New-Hampshire adulte, aucun accident mortel ne se produit pour des doses allant jusqu'à $1 \mathrm{~g} / \mathrm{kg}$. La dose de $1,5 \mathrm{~g} / \mathrm{kg}$ tue deux poulets sur quatre : la dose active étant de $100 \mathrm{mg} / \mathrm{kg}$, KERR pense que le coefficient chimiothérapique est proche de 20.

En 1959, ENIGK etDUWEL notent que la dose de $470 \mathrm{mg} / \mathrm{kg}$ est fatale à un poulet sur six et que la dose de $564 \mathrm{mg} / \mathrm{kg}$ tue tous les animaux mis en expérience.

Ces résultats sont évidemment très discordants, aussi, nous avons repris complètement cette étude.

Les tests de toxicité effectués à Fort-Lamy donnent les résultats suivants (Voir tableau no VIII).

(*) Docteur SALSBURY'S Laboratories. Charles CITY lowa. U.S. A.

(**) Omnivermyl: Laboratoires Veteravic. Paris.

\begin{tabular}{|c|c|c|c|}
\hline \multicolumn{4}{|c|}{ TABLEAU VIII } \\
\hline $\begin{array}{c}\text { Doses } \\
\text { (mg/kg) }\end{array}$ & $\begin{array}{c}\text { Nombre d' } \\
\text { animaux }\end{array}$ & $\begin{array}{c}\text { Nombre de } \\
\text { morts }\end{array}$ & $\begin{array}{c}\text { Pourcentage } \\
\text { de mortalité }\end{array}$ \\
\hline 50 & 3 & néant & \\
80 & 6 & nézrnt & 3 \\
100 & 30 & 1 & 3 \\
125 & 7 & 1 & 14 \\
150 & 10 & 1 & 10 \\
180 & 11 & néant & \\
200 & 16 & 4 & 25 \\
250 & 6 & 3 & 50 \\
300 & 3 & néant & 5 \\
400 & 2 & 1 & 50 \\
500 & 9 & 3 & 33 \\
600 & 3 & 2 & 66 \\
1000 & 6 & 4 & 66 \\
1200 & 3 & 1 & 33 \\
1500 & 6 & 4 & 66 \\
\hline
\end{tabular}

Nos résultats sont en complète contradiction avec ceux obtenus par KERR. II nous parut alors important de recommencer les essais de KERR en utilisant la même race de poulet que cet auteur. Nous avons pour cela déterminé d'une manière précise: la $\mathrm{DL} 50$ sur' des poulets NewHampshire à Montpellier.

Cette détermination a été faite de la manière suivante:

120 poussins New-Hampshire âgés de 8 jours ont été placés en batterie au laboratoire et élevés dans des conditions identiques. La nourriture était essentiellement constituée par un régime équilibré synthétique. Après 14 semaines, on a trié 80 poulets pesant $1,500 \mathrm{~kg} \pm 150 \mathrm{~g}$.

Nous avons administré le DED en capsules de gélatine strictement dosées. Les animaux ont été maintenus à la diète 12 heures avant le traitement et 6 heures après. Les doses administrées à 70 poulets répartis en 7 lots de 10 sont respectivement $0,8 \mathrm{~g}, 1 \mathrm{~g}, 1,2 \mathrm{~g}, 1,4 \mathrm{~g}, 1,6 \mathrm{~g}, 1,8 \mathrm{~g}, 2 \mathrm{~g}$ par kg d'animal, 10 poulets non traités servent de témoins.

Les composés organiques de l'étain étant susceptibles de manifester une toxicité différée (CAUJOLLE et coll. 1954, MEYNIER 1956, 1957), la DL 50 a été calculée après avoir maintenu les animaux en observation pendant 6 semaines.

Le temps de crise se situe entre le troisième ef lé huitième jour après l'administration du toxique ; passé ce temps, nous n'avons observé que deux cas de mort retardée, le $17 \mathrm{e}$ et le $18 \mathrm{e}$ jour, dans le groupe ayant reçu $2 \mathrm{~g} / \mathrm{kg}$.

Les résultats sont rapportés dans le tableau IX. La DL 50 calculée par la méthode de BLISS FINNEY est de $1,66 \mathrm{~g} / \mathrm{kg}$ avec un intervalle de confiance 1,31-1,90 pour le niveau de probabilité 


\begin{tabular}{|c|c|c|c|c|c|c|c|}
\hline \multicolumn{8}{|c|}{ MABLEAU IX } \\
\hline \multicolumn{2}{|c|}{ Lot I } & \multicolumn{2}{|c|}{ Lot II } & \multicolumn{2}{|c|}{ Lot III } & \multicolumn{2}{|c|}{ Lot IV } \\
\hline $\begin{array}{r}\text { Témo: } \\
\text { vivants } \\
10\end{array}$ & $\begin{array}{c}\text { ins } \\
\text { morts } \\
0\end{array}$ & $\begin{array}{c}800 \\
\text { vivants } \\
10\end{array}$ & $\begin{array}{c}\mathrm{mg} / \mathrm{kg} \\
\text { morts } \\
0\end{array}$ & $\begin{array}{c}1 E \\
\text { vivants } \\
8\end{array}$ & $\begin{array}{c}\text { mort.s } \\
2\end{array}$ & $\begin{array}{r}1200 \\
\text { vivants } \\
7\end{array}$ & $\mid \begin{array}{c}\mathrm{mg} / \mathrm{kg} \\
3\end{array}$ \\
\hline \multicolumn{2}{|c|}{ Lot V } & \multicolumn{2}{|c|}{ Lat VI } & \multicolumn{2}{|c|}{ Lot VII } & \multicolumn{2}{|c|}{ Lot VIII } \\
\hline $\begin{array}{c}1400 \mathrm{mat} \\
\text { Whants } \\
7\end{array}$ & $\mid \begin{array}{c}\text { morts } \\
3\end{array}$ & $\begin{array}{c}1600 \mathrm{me} \\
\text { vivants } \\
6\end{array}$ & $\begin{array}{c}\text { ngtkg } \\
\text { morts } \\
4\end{array}$ & \multicolumn{2}{|c|}{\begin{tabular}{c|c}
\multicolumn{2}{c}{$1800 \mathrm{mg} / \mathrm{kg}$} \\
vivants & morts \\
5 & 5
\end{tabular}} & \multicolumn{2}{|c|}{$\begin{array}{c}2 \mathrm{~g} / \mathrm{kg} \\
\text { vivants / Forts }\end{array}$} \\
\hline
\end{tabular}

$P=0,05$. On voit que dans ces conditions on obtient des résultats qui sont en accord avec ceux de KERR. Il faut donc déduire de ces essais que la toxicité du DED varie d'une espèce de poulet à une autre, cette variation est très importante en ce qui concerne le poulet africain.

Comment expliquer cette sensibilité anormale du poulet africain au DED ? Nous avons établi un tableau donnant la mortalité en fonction de l'époque où a eu lieu le traitement (tableau $X$ ).

Deux cas doivent être envisagés :

a) Cos exceptionnel : les poulets sont en excellent état, comme en septembre 1961. Bien nourris, du fait de la saison, peu anémiés, leur résistance est bonne. Les premiers décès n'apparaissent alors qu'à $400 \mathrm{mg} / \mathrm{kg}$. Même à des doses plus fortes, tous les poulets ne meurent pas : pas de mortalité d̀ $500 \mathrm{mg} / \mathrm{kg}$, mortalité de $2 / 3$ à $800 \mathrm{mg} /$ $\mathrm{kg}$ ef de $1 / 3$ à 1.200 et $1.500 \mathrm{mg} / \mathrm{kg}$. Dans les mêmes conditions et aux mêmes doses, certains animaux sont donc plus sensibles à l'action de l'anthelminthique que d'autres. Dans ce cas, le facteur individuel semble jover un rôle particulièrement important.

b) Cos le plus fréquent : les poulets proviennent de lots variés qui renferment des animaux en bon état et d'autres en moins bon état, plus ou moins anémiés, ainsi que le démontrent les nombreuses numérations globulaires effectuées en juin-juillet 1960 et en février-mars-avril 1961. Les premiers accidents mortels se manifestent déjà à $100 \mathrm{mg}$ ' $\mathrm{kg}$, et, à la dose thérapeutique indiquée, on risque - mais ce n'est pas un phénomène absolu de voir mourir de 10 à 25 p. 100 des animaux troités.

Comme il est très rare d'avoir affaire constamment à des animaux en excellent état, que les lots sont la plupart du temps médiocres, et que l'on est appelé à déparasiter les animaux même en période défavorable, le DED se révèle d'un emploi délicat; les doses thérapeutiques et les doses toxiques se chevauchent en partie. De plus, si les doses sont trop fortes, les risques de mortalité augmentent ; si elles sont trop faibles, certains Cestodes ne sont pas détruits et un grand nombre de formes immatures persistent.

L'intoxication par le DED se traduit par :

- Soit des signes de paralysie, le poulet est accroupi et ne peut se relever. S'il se déplace il le fait très lentement «en canard». Le cou est tordu sur lui-même. Dans d'autres cas, la tête est animée d'un lent mouvement de balancier. On note du nystagmus. L'appétit disparaît.

- Soit par une apathie totale, le poulet a la tête dans les ailes et demeure indifférent à tout ce qui se passe à l'extérieur. L'amaigrissement est rapide et les numérations globulaires pratiquées pendant les 3 jours qui suivent le traitement indiquent une diminution considérable des hé-

\begin{tabular}{|c|c|c|c|}
\hline \multicolumn{4}{|c|}{ TABLEAU $X$} \\
\hline $\begin{array}{r}\text { Doses } \\
(\mathrm{mg} / \mathrm{kg})\end{array}$ & Mortalité & Epoque des traitenents & $\begin{array}{l}\text { Etat'des } \\
\text { animaux }\end{array}$ \\
\hline 100 & $\begin{array}{lll}0 & \text { sur } & 6 \\
1 & \text { sur } & 20 \\
0 & \text { sur } 4\end{array}$ & $\begin{array}{l}\text { Juin - Jujllet } 1960 \\
\text { Mars - Avril } 1961 \\
\text { Septembre } 1961\end{array}$ & $\begin{array}{l}\text { moyen } \\
\text { tres moyen } \\
\text { excellent }\end{array}$ \\
\hline 125 & $\begin{array}{l}1 \text { sur } 4 \\
0 \text { sur } 3\end{array}$ & $\begin{array}{l}\text { Février } 1961 \\
\text { Septembre } 1961\end{array}$ & $\begin{array}{l}\text { trìs moyon } \\
\text { excellent }\end{array}$ \\
\hline 150 & $\begin{array}{l}1 \operatorname{sur} 7 \\
0 \operatorname{sur} 3\end{array}$ & $\begin{array}{l}\text { Pévrier } 1961 \\
\text { Septembre } 1961\end{array}$ & $\begin{array}{l}\text { très ' noyei: } \\
\text { excellent }\end{array}$ \\
\hline 200 & $\begin{array}{lll}1 & \text { sur } & 4 \\
3 & \text { sur } & 9 \\
0 & \text { sur } & 3\end{array}$ & $\begin{array}{l}\text { Juin - Juillet } 1960 \\
\text { Février - Mars } 1961 \\
\text { Septembremoctobre } 1961\end{array}$ & $\begin{array}{l}\text { moyen } \\
\text { très moyen } \\
\text { ercellent }\end{array}$ \\
\hline 250 & $\begin{array}{l}1 \text { sur } 3 \\
2 \text { sur } 3\end{array}$ & $\begin{array}{l}\text { Juin - Juillet } 1960 \\
\text { Pévrier } 1961\end{array}$ & moyen \\
\hline 300 & 0 sur 3 & Juin - Juillet 1960 & moyen \\
\hline 400 & 1 sur 2 & Septembre 1961 & excellent \\
\hline 500 & $\begin{array}{l}1 \text { sur } 3 \\
2 \text { sur } 3 \\
0 \text { sur } 3\end{array}$ & $\begin{array}{l}\text { Juin - Juillet } 1900 \\
\text { Mars - Avril 1961 } \\
\text { Septembre } 1961\end{array}$ & $\begin{array}{l}\text { moyen } \\
\text { très moyen } \\
\text { excellent }\end{array}$ \\
\hline 800 & 2 sur 3 & Septembre 1961 & excellent \\
\hline 1000 & $\begin{array}{l}3 \operatorname{sur} 3 \\
1 \operatorname{sur} 3\end{array}$ & $\begin{array}{l}\text { Hars - Avril } 1961 \\
\text { Septembre } 1961\end{array}$ & $\begin{array}{l}\text { très moyen } \\
\text { excell lent }\end{array}$ \\
\hline 1200 & 1 sur 3 & Septembre 1961 & très moyen \\
\hline 1500 & $\begin{array}{l}3 \text { sur } 3 \\
1 \text { sur } 3\end{array}$ & $\begin{array}{l}\text { Mars - Avril } \\
\text { Octobre } 1961\end{array}$ & $\begin{array}{l}\text { très moyen } \\
\text { excellent }\end{array}$ \\
\hline $\begin{array}{l}125 \\
(\text { par } \\
\text { tête) }\end{array}$ & 0 sur 11 & Octobre 1961 & excellent \\
\hline
\end{tabular}


maties (2.500.000 globules rouges 3 jours après le traitement).

Enfin, un des signes d'intoxication les plus constants est une diarrhée importante qui débute 4 heures après l'administration du toxique et qui se prolonge 4 à 5 jours suivant la dose absorbée.

Du point de vue anatomo-pathologique les principales lésions ont été observées dans le foie. Nous avons observé sur des poulets ayant reçu de 250 à $1.500 \mathrm{mg} / \mathrm{kg}$ de DED et morts d'intoxication 4 à 5 jours après le traitement, des lésions hépatiques importantes se traduisant par une hépatite nécrosante pluri-lobulaire. I e foie est de couleur feville morte ef très friable. II est intéressant de remarquer que dans le test de toxicité effectué sur des poulets New-Hampshire, 20 poulets survivants sur 46 étaient porteurs de lésions hépatiques. Ces lésions sont caractérisées par des foyers plus ou moins bien circonscrits d'hépatite nécrosante intéressant plusieurs lobules.

\section{$2^{\circ}$ Toxicité pour l'homme des viandes traitées.}

Si d'une façon gćnćralc, los animaux ayant subi un traitement, ne sont pas, en principe, destinés à une consommation immédiate, il n'en est pas de même dans certains pays. Au Tchad par exemple, il est courant de voir abattre pour les consommer, des animaux prćscntant des signes de faiblesse ou de maladie, voire des signes d'intoxication à la suite d'un traitement.

D'autre part, les composés organiques de l'étain étant beaucoup plus toxiques pour les mammifères que pour les oiseaux, il paraît particulièrement important de ne pas négliger cet aspect de la toxicité du DED.

La DL 50 du DED est pour le rat, de $146 \mathrm{mg} /$ $\mathrm{kg}\left({ }^{*}\right)$. L'un de nous a d'ailleurs indiqué dès 1956 , que ce composé était inutilisable comme anlhelminthique chez les mammifères, la dose active provoquant déjà des signes d'intoxication.

La toxicité aiguë et chronique des composés dibutyl et tributylétain a été étudiée en détail par BARNES et STORNER $(1958,1959)$ et par BARNES et MAGGEE (1958).

Les sels de dibutylétain provoquent chez le rat des lésions caractéristiques des canaux biliaires et une dégénérescence fibreuse du pancréas. Une

(*) G. GRAS, thèse Pharmacie 1956 : Communication personnelle du Docteur K. B. KERR. dose unique de $20 \mathrm{mg} / \mathrm{kg}$ ou de $50 \mathrm{ppm}$ de dichlorure d'étain dibutyle, donnée dans la nourriture pendant 6 mois, suffit pour faire apparaître ces lésions. La plus forte concentration inoffensive supportée par le rat est de 20 ppm. Dans ces conditions BARNES et STORNER pensent que si l'on applique le « 100 fold safety factor 》 les résidus de dibutylétain dans la nourriture ne doivent pas excéder 0.2 ppm.

La recherche des résidus de DED a été faite par le dosage de l'étain dans des organes de poulets traités.

Nous avons opéré de la manière suivante :

9 poulets New-Hampshire pesant $2.000 \mathrm{~g} \pm$ $100 \mathrm{~g}$ reçoivent une dose unique de $125 \mathrm{mg} / \mathrm{kg}$; cette dose, qui représente la dose thérapeutique standard est administrée en capsules après diète de 12 heures. Les poulets sont sacrifiés 3 jours, 6 jours et 9 jours après le traitement. La viande et les organes sont prélevés immédiatement et pesés. On procède ensuite à la destructión de la matière organique par la méthode sulfo-nitro-perchlorique. Nous avons détruit des qu'antités de matières organiques assez importantes de manière à ce que les résultats qui sont exprimés'en mg d'étain par $\mathrm{kg}$ de tissus frais reflètent d'une manière aussi valable que possible la répartition du toxique. Ceci n'est pas toujours le cas lorsqu'on' se contente d'opérer sur des parties aliquotes de 10 à $15 \mathrm{~g}$. Dans ce but, le foie, les reins ef le gésier sont détruits en entier, pour l'aile et la cuisse les quantités de matières organiques détruites sont comprises entre 50 et $60 \mathrm{~g}$.

L'étain est séparé par distillation sous forme de bromure et dosé par la méthode spectrophotométrique au dithiol d'OVENSTON ef KENYON (1955). Cette méthode permet de doser jusqu'à $5 \mu \mathrm{g}$ dans la prise d'essai avec une erreur relative qui n'excède pas 10 p. 100 . Une technique à peu près identique a été décrite récemment par BURGER (1961).

Sur les muscles, les dosages de contrôles ont été effectués par la méthode polarographique de GODARD et ALEXANDER (1945).

Les résultats sont rapportés dans le tableau $X I$.

Ces résultats montrent que même pour la dose thérapeutique, des quantités non négligeables de DED sont absorbées. Toutefois, ces quantités sont éliminées complètement au bout de 9 jours: En effet, dans le groupe sacrifié 9 jours après le traitement, on n'a pas trouvé de trace d'étain. 
Par conséquent, les poulets ayant subi un traitement par le DED peuvent être consommés sans danger 8 à 9 jours après le traitement.

Toutefois, lorsque le DED est administré mélangé à la nourriture les quantités résorbées dans l'intestin sont beaucoup plus importantes. C'est ainsi qu'après administration pendant 13 jours de nourriture contenant du DED dans les proportions de 0,10 p. $100,0,05$ p. 100 et 0,0375 p. 100 , $\operatorname{KERR}\left({ }^{*}\right)$ a trouvé des quantités relativement

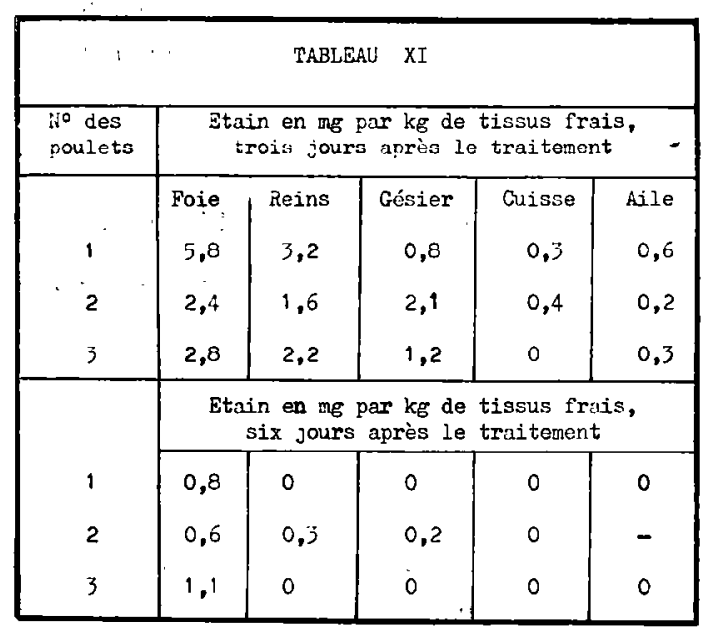

importantes d'étain dans le foie. Les résultats sont rapportés dans le tableau no XII.

De même, des dindons qui reçoivent du DED mélangé à la nourriture dans la proportion de 0,5 p. 100 pendant 4 semaines ont, dans le foie, après ce temps, des concentrations en étain de $15 \mathrm{mg} / \mathrm{kg}$.

\begin{tabular}{|c|c|c|}
\hline \multicolumn{3}{|c|}{ TABLEASU XII } \\
\hline $\begin{array}{l}\text { Concentration de DED } \\
\text { dans la nourriture }\end{array}$ & $x^{\circ}$ des poules & $\begin{array}{l}\text { Etain en mo/kg } \\
\text { après lis jours }\end{array}$ \\
\hline $\begin{aligned} \text { Groupe } n^{\circ} & 1 \\
0,10 \text { p. } & 100\end{aligned}$ & $\begin{array}{l}5.561 \\
5.685 \\
5.609\end{array}$ & $\begin{array}{l}11,8 \\
15,5 \\
18,7\end{array}$ \\
\hline $\begin{array}{l}\text { Groupe } n^{\circ} 2 \\
0,05 \text { p. } 100\end{array}$ & $\begin{array}{l}5.654 \\
5.656 \\
5.624 \\
5.686\end{array}$ & $\begin{array}{l}7.67 \\
7,52 \\
5,72 \\
6,99\end{array}$ \\
\hline $\begin{array}{l}\text { Groupe } n^{0} 3 \\
0,0375 \text { p. } 100\end{array}$ & $\begin{array}{l}5.615 \\
5.617 \\
5.622 \\
5.638\end{array}$ & $\begin{array}{l}5,94 \\
8,55 \\
5,71 \\
6,90\end{array}$ \\
\hline
\end{tabular}

Il semble donc, que des précautions supplémentaires doivent être prises lorsque le DED est administré d'une manière continue dans la nourriture.

Enfin, il faut remarquer que la méthode qui consiste à doser l'étain et non le composé organique en bloc est imparfaite, car cette manière d'opérer n'indique pas sous quelle forme se trouve l'étain dans l'organisme.

Or, du point de vue toxicologique, seul le grou: pe dibutylétain est dangereux. II sera donc très important de pouvoir préciser ce point. Plusieurs méthodes spécifiques de dosages ont déjà été publiées (ALDRIGE et CREMER 1957 CHAPMAN, DUCKWORTH ef PRICE, 1959, BURGER 1960) il est probable que l'utilisation d'une de ces méthodes associée à une technique d'extraction et de séparation convenable, comme par exemple les méthodes chromatographiques récemment décrites (BARBIERI, 1958 ; WILLIAMS et PRICE, 1960, BRUGGEMAN 1959), permettra de préciser sous quelle forme le DED se trouve dans l'organisme du poulet.

\section{VII. - CONCLUSIONS}

Le Dilaurate d'étain dibutyle fait preuve d'une bonne activité anthelminthique sur les formes adultes de Choanotaenia infundibulum, Raillietina tetragona, Raillietina echinobothrida, Raillietina cesticillus, Cotugnia digonopora, Hymenolepis carioca. Les formes immatures de ces mêmes parasites sont beaucoup plus résistantes ef 5 à 10 p. 100 d'entre elles persistent à la dose de $125 \mathrm{mg} / \mathrm{kg}$ qui est la plus couramment utilisée. L'élimination de Davainea proglottina et d'Amoebotaenia sphenoïdes paraît exiger des doses plus fortes. Pour la dose thérapeutique standard de $125 \mathrm{mg} / \mathrm{kg}$, de petites quantités de DED sont résorbées par la muqueuse intestinale; les plus fortes concentrations se trouvent dans le foie ; le produit est complètement éliminé 6 à 9 jours après le traitement.

Dans les conditions africaines, où il faut tenir compte de la cohabitation possible des formes adultes et des formes immatures de divers Cestodes à toutes les époques de l'année, il est conseillé d'administrer $125 \mathrm{mg}$ par tête à des poulets dont le poids n'est guère supérieur à $1.100 \mathrm{~g}$. 
Le produit est donné soit en capsules de gélatine, soit mélangé à de la nourriture dans des proportions variables.

La diète de 20 heures avant ef de 3 heures après le traitement est recommandée.

Malheureusement, si le DED est assez bien toléré par des animaux en excellent état, lorsqu'on a affaire à des poulets maigrès, anémiés et parasités ce quil constitue l'essentiel des lots soumis au traitement, à des doses voisines de la dose thérapeutique $(150-200 \mathrm{mg} / \mathrm{kg})$, des accidents mortels apparaissent : ils peuvent toucher le quart des animaux en traitement. Les facteurs individuels semblent jouer le rôle important dans. la sensibilité du poulet africain au DED.

C'est pourquoi, dans les pays tropicaux, cet anthelminthique doit être employé avec la plusi extrême prudence.

\section{Laboratoire de recherches vétérinairés de Forcha - Fort-Lamy (Tchad) \\ (Service de parositologie) \\ Faculté de pharmacie de Montpellier (Service de pharmacie chimique).}

\section{SUMMARY}

\section{Studies on the anthelmintic activity and toxicity of the Dilaurate of tin dibutyle (DED) in poultry}

This chemical is shown to be of good anthelmintic value on the adult forms of Choanotaenia infundibulum, Raillietina tetragona, Raillietina echinobothrida, Raillietina cesticillus, Cotugnia digonopora, Hymenolepis carioca. The immature forms of these species are much more resistant and up to $10 \mathrm{p} .100$ persist at the usual dosage of $125 \mathrm{mg} / \mathrm{kg}$. The elimination of Dovainea proglottina and Amoebotaenia sphenoides requires heavier dosage. With the standard dose of $125 \mathrm{mg} / \mathrm{kg}$ of DED small quantities are absorbed by the intestinal mucosa; the strongest concentrations being found in the liver but is completely eliminated 6 to 9 days after treatment.

Under African conditions where the presence of adult and immature forms of Cestodes may be found together at ali seasons of the year, it is advisable to dose poultry up to $11 / 2 \mathrm{lb}$ in weight with $125 \mathrm{mg}$. The product may be given in gelatine capsules or mixed with the food. Fasting for 20 hours before and 3 hours after treatment is recommended.

Unfortunately while DED is well tolerated by birds in good condition, treatment of birds which are emaciated, anaemic and parasited (which is usually the case) in doses around 150-200 mg/kg will result in death of up to $25 \mathrm{p}$. 100 of treated birds - Idiosyncrasy' to the drug is an 'important factor in African poultry - In tropical countries at least the drug should be used with extreme: prudence.

\section{RESUMEN}

Estudios respecto a la actividad antihelmintica y de la toxicidad del dilaurato de estaño dibutilo en el pollo

El Dilaurato de estaño Dibutilo ha' demostrado poseer una buena actividad antihelmíntica sobre las formas adultas de Choanotaenia infundibulum, Raillietina tetragona, Raillietina echinobothrida, Raillietina cestillus, Cotugnia digonopora y Hymenolepis carioca. Las formas inmaturas de estos mismos parasitos son mucho más resistentes y un 5 a un 10 por ciento de los mismos siguen persistiendo una vez aplicada una dosis de $125 \mathrm{mg} / \mathrm{kg}$ que es aquella que se utiliza más corrientemente. La eliminación de Davainea proglottina y de Amoebotaenia'sphenoides parece requerir dosis mayores. Pará la dosis terapéutica normal de $125 \mathrm{mg} / \mathrm{kg}$, una pequeña proporción de DED es reabsorbida por la mucosa intestinal. Las mayores concentraciones se encuentran en el higado. El producto queda completamente eliminado transcurridos 6 a 9 dias después del tratamiento.

En las condiciones africanas, en las cuales cabe tener en cuenta la cohabitación posible de las formas aduiltas y de las formas inmaturas de diversos Cestodos en todas las épocas del año, se aconseja administrar $125 \mathrm{mg}$ por cabeza a los pollos cuyo peso no sobrepase de $1.100 \mathrm{~g}$. 
El producto es dado en cápsulas de gelatina o bien mezclado con los alimentos, en proporciones variables.

Se recomienda hacer observar una dieta de 20 horas antes y 3 horas después del tratamiento.

Desdichadamente, si bien el DED es bastante bien tolerado por los animales en excelente estado de salud, cabe tener en cuenta que en el caso de pollos flacos, anemiados y muy contaminados por los parásitos, cáso que se presenta en las partidas sometidas al tratamiento con bastante frecuencia, se tropieza con casos normales cuando se aplican dosis cercanas de la dosis terapeutica (150$200 \mathrm{mg} / \mathrm{kg}$ ). Estos accidentes pueden alcanzar a la cuarta parte de los animales en trałamiento. Los factores individuales parecen desempeñar un papel importante en la sensibilidad del pollo africano tratado con DED.

Por estas razones, este antihelmíntico debe ser empleado con la mayor prudencia cuando se trata de los paises tropicales.

\section{BIBLIOGRAPHIE}

(1) $\operatorname{ABDOU}($ A. H.). - J. Egypt. Public Heolth. Ass. 32 (3), 151-165.

(2) ALDRIDGE (W. N.) et CREMER (J. E.). Analyst, 1957, $82(970): 37-43$.

(3) BARBIERI (R.), BELLUCO (U.) ef TAGLIAVINI (G.). - Ann. Chim. Romo, 1958, $48: 940$.

(4) BARNES (J.M.) et MAGEE (P. N.). - J. Poth. Bact. 1958, 75 (2), 276-279.

(5) BARNES (J. M.) et STORNER (H. B.). Brit. J. Industr. Méd. GB, 1958 (1), 15-22.

(6) BARNES (J. M.) et STORNER (H. B.). 1959. Pharmacol, rev. 11, 211-231.

(7) BLOUNT (W. P.). - 1955, vet. rec. 67 (504), 1095.

(8) BRUGGEMAN (J.). - Testimonial of 2. 2. 1959.

(9) BURGER (K.). - Z. Lebensm - Untersuch. U. Forsch, 1961, II 4 (1), 10-13.

(10) BURGER (K.). - Ztschr.f. Lebensmittel -Untersuchung und - Forschung. 1961, I14 (1), 1-10.

(11) CASTEL (P.), GRABER (M.). GRAS (G.) et CHHAY - HANCHENG. - 1960, Rev. Elev. Méd. vét. Pays trop., 13 (4), 281-296.

(12) CASTEL (P.), GRABER (M.). GRAS (G.) ef CHHAY - HANCHENG. - 1960, Rev. Elev. Méd. vét. Pays trop., 13 (1), 57-74.

(13) CAUJOLLE (F.), LESBRE (M.) et MEYNIER (D.). - Acod. Sc., 1954, 239 (7), 556.

(14) CAUJOLLE (F.), LESBRE (M.) et MEYNIER (D.). - Acad. Sc. 1954, 239 (17), 1091.
(15) CHAPMAN (A. H.), DUCKWORTH (M. W.) et PRICE (J. W.). - British Plastics, 1959, 296-299.

(16) EDGAR (S. A.). - 1956, Poult. Sci. 35, 64-73.

(17) $\operatorname{EDGAR}$ (S. A.) et TEER (P. A.). - 1957. Poult. Sci. 36, 329-334.

(18) ENIGK (K.) et DUWEL (D.). - 1959, Deutsche. Tierazz. Woch., 66 (1), 10-16.

(19) GODARD (M. E.) et ALEXANDER (O. R.).-Ind. Eng. Chem. Anal. Ed., 1946, 18, 681-689.

(20) GRAS (G.). - Thèse Pharm. Montpellier, juillet 1956.

(21) GUILHON (J.) et GRABER (M.). - Rev. Elev. Méd. vét. Pays trop. 1960, 13 (4), 297-304.

(22) GUILHON (J.) et GRABER (M.). - Rev. Elev. Méd. vét. Poys trop., 1961, I4 (1), 57-65.

(23) GUILHON (J.) ef GRABER (M.). - Bull. Acad. vét., 1961, 34 (6), 241-243.

(24) GUILHON (J.) etGRABER (M.). - Bull. Acad. vet., 1961, 34 (5), 187-192.

(25) GUTHRIE (J. E.) et HARWOOD (P. D.). 1950, Amer. J. vét. Res. I (1), 108-116.

(26) GUTHRIE (J. E.), POWICK (W.C.) et BLANDELL (D.). - North. Amer. vet. 1941, 22, 2224.

(27) KERR (K. B.). - 1952, Poult. Sci. 31, 236-238,

(28) KERR (K. B.) et WALDE (A. W.). - 1951, J. Parasit. 37 (5), Suppl. 2, 27-28.

(29) KERR (K. B.) et WALDE (A. W.). - 1956. Exp. Parasit. 5, 560-570. 
(30), KOCHESKKOV (K. A.). - Ber. Dtsch, Chem. Gesellsch, 1928, 6I, (8), 1659-1663.

(31) MEYNIER (D.). - Thèse doct. Sciences, Toulouse, 1956.

(32) MEYNIER (D.). - Acad. Sc; 1957, 245 (25), 2428-2430.

33) OVENSTONE (T.C. J.) et KENYON (C.). Anolyst, 1955, 80, 566-567.
(34) REID (W.M.) et NUGARA (D.). - 1959, J. Parasit. 45, suppl., 45.

(35) SMITH (H. W.). - 1952, Plastics, 17, 264. (36) TURK (R. D.). - 1958, J. Amer. vet. Med. Ass. I32, 13-15.

(37) WILLIAMS (D. J.), et PRICE (J.W.). - Analyst, 1960, 85 (1.013), 579-582.

(38) WHITTEN (L. K.). - 1956, vet. Rev. Ann. 2 (1) 18. 